International Mathematical Forum, 2, 2007, no. 4, 195 - 201

\title{
A note on the existence and stability properties of positive solution for some Dirichlet problems
}

\author{
G. A. Afrouzi and S. H. Rasouli \\ Department of Mathematics, Faculty of Basic Sciences \\ Mazandaran University, Babolsar, Iran \\ afrouzi@umz.ac.ir
}

\begin{abstract}
This study concerns the existence and stability properties of positive solutions to classes of boundary value problems of the form

$$
\left\{\begin{array}{clrl}
-\Delta u & =\lambda a(x) f(u)-c, & & x \in \Omega, \\
u & =0, & & x \in \partial \Omega,
\end{array}\right.
$$

where $\lambda, c>0$ are parameters, $\Delta$ is the Laplacian operator, $\Omega$ is a bounded domain in $R^{N}(N>1)$ with smooth boundary $\partial \Omega$, the weight $a(x)$ satisfies $a(x) \in C(\Omega), a(x) \geq a_{0}>0$ for all $x \in \Omega$, and $f$ is a $C^{2}([0, \infty))$ function such that $f(0)=0$. First by using the method of sub-super solution we study the existence of positive solution under the assumption that $f(u)>0$ for $0<u<\rho$ and $f(u) \leq 0$ for $u \geq \rho$. Next we study the stability properties of positive solution under the assumption that $t \mapsto \frac{f(t)}{t}$ is strictly increasing.
\end{abstract}

keywords: positive solutions, sub-super solution, linearized stability

Mathematics Subject Classification: 35J55, 35K60

\section{Introduction}

In this paper we consider the existence and stability properties of positive solution to classes of boundary value problems of the form

$$
\begin{gathered}
-\Delta u=g(x, u) \equiv \lambda a(x) f(u)-c, \quad x \in \Omega, \\
u=0, \quad x \in \partial \Omega,
\end{gathered}
$$

where $\lambda, c>0$ are parameters, $\Delta$ is the Laplacian operator, $\Omega$ is a bounded domain in $R^{N}(N>1)$ with smooth boundary $\partial \Omega$, the weight $a(x)$ satisfies 
$a(x) \in C(\Omega), a(x) \geq a_{0}>0$ for all $x \in \Omega$, and $f$ is a $C^{2}([0, \infty))$ function such that $f(0)=0$.

First we study the existence of positive weak solution under the the following assumptions:

(H1) $f \in C^{2}([0, \infty)), f(0)=0, f(u)>0$ for $0<u<\rho$ and $f(u) \leq 0$ for $u \geq \rho$ for some $\rho>0$.

When $c=0$ it is easy to establish the existence of a positive solution for large $\lambda>0$. Here we consider the challenging semipositone case $c>0$.

Semipositone problems arise in buckling of mechanical systems, design of suspension bridges, chemical reactions, astrophysics (thermal equilibrium of plasmas), combustion and management of natural resources.

As pointed out by P.L. Lions in [7], semipositone problems are mathematically very challenging, and from the point of view of many important natural applications, interesting, particularly for positive solutions. In fact, during the last ten years finding positive solutions to problems of the form (1)-(2) with $g(x, 0)<0$ has been actively pursued. The difficulty of studying positive solutions to such problems was first encountered by Brown and Shivaji in [3] when they studied the perturbed bifurcation problem

$$
\begin{array}{r}
-\Delta u=\lambda\left(u-u^{3}\right)-\epsilon, \quad x \in \Omega, \\
u=0, \quad x \in \partial \Omega,
\end{array}
$$

with $\epsilon>0$. In general, studying positive solutions for semipositone problems is more difficult compared to that of positone problems. The difficulty is due to the fact that in the semipositone case, solutions have to live in regions where the reaction term is negative as well as positive. We will briefly introduce the method of sub-super solutions (see $[5,8]$ ), which has been a very successful method in handling positone problems, to demonstrate the difficulty of studying positive solutions for semipositone problems over positone problems.

A super solution to (1)-(2) is defined as a function $z \in C^{2}(\bar{\Omega})$ such that

$$
\begin{array}{r}
-\Delta z \geq \lambda g(x, z) \quad x \in \Omega, \\
z \geq 0, \quad x \in \partial \Omega .
\end{array}
$$

Sub solutions are defined similarly with the inequalities reversed and it is well known that if there exists a sub solution $\psi$ and a super solution $z$ to (1)-(2) such that $\psi(x) \leq z(x)$ for $x \in \bar{\Omega}$, then (1)-(2) has a solution $u$ such that $\psi(x) \leq u(x) \leq z(x)$ for $x \in \bar{\Omega}$. Further note that if $\psi(x) \geq 0$ for $x \in \Omega$ then $u \geq 0$ for $x \in \Omega$.

In [2], when $c$ is very small the authors establish an existence of a positive solution for $\tilde{\lambda}$ near the first eigenvalue $\lambda_{1}$ and then extend the existence for $\lambda \geq$ 
$\tilde{\lambda}$. In this paper, we establish the existence of a positive solutions directly for $\lambda$ large. We refer to $[1,2,4,6]$ for additional results on semipositone problems.

We next study the stability of positive weak solutions under the additional assumption $t \mapsto \frac{f(t)}{t}$ is strictly increasing.

\section{Existence results}

In this section we establish positive constants $\left.c_{0}(\Omega, \rho)\right)$ and $\left.\lambda_{0}(\Omega, \rho, c)\right)$ such that the problem $(1)-(2)$ has a positive solution when $c \leq c_{0}$ and $\lambda \geq \lambda_{0}$. We employ the method of sub-super solutions to obtain the result ( see $[5,8]$ ).

To precisely state our existence result we consider the eigenvalue problem

$$
\begin{cases}-\Delta \phi=\lambda \phi, & x \in \Omega, \\ \phi=0, & x \in \partial \Omega .\end{cases}
$$

Let $\phi_{1} \in C^{1}(\bar{\Omega})$ be the eigenfunction corresponding to the first eigenvalue $\lambda_{1}$ of (3) such that $\phi_{1}(x)>0$ in $\Omega$, and $\left\|\phi_{1}\right\|_{\infty}=1$. It can be shown that $\frac{\partial \phi_{1}}{\partial n}<0$ on $\partial \Omega$ and hence, depending on $\Omega$, there exist positive constants $k, \eta, \mu$ such that

$$
\begin{gathered}
\lambda_{1} \phi_{1}^{2}-\left|\nabla \phi_{1}\right|^{2} \leq-k, \quad x \in \bar{\Omega}_{\eta}, \\
\phi_{1} \geq \mu, \quad x \in \Omega_{0}=\Omega \backslash \bar{\Omega}_{\eta},
\end{gathered}
$$

with $\bar{\Omega}_{\eta}=\{x \in \Omega \mid d(x, \partial \Omega) \leq \eta\}$.

We will also consider the unique solution, $\zeta \in C^{1}(\bar{\Omega})$, of the boundary value problem

$$
\begin{cases}-\Delta \zeta=1, & x \in \Omega, \\ \zeta=0, & x \in \partial \Omega,\end{cases}
$$

to discuss our existence result. It is known that $\zeta>0$ in $\Omega$ and $\frac{\partial \zeta}{\partial n}<0$ on $\partial \Omega$. Our existence result is formulate in the following theorem.

Theorem 2.1. Let $(H 1)$ hold. Then there exists positive constants $c_{0}=$ $\left.c_{0}(\Omega, \rho)\right)$ and $\left.\lambda_{0}=\lambda_{0}(\Omega, \rho, c)\right)$ such that the problem $(1)-(2)$ has a positive solution when $c \leq c_{0}$ and $\lambda \geq \lambda_{0}$.

Proof. First we construct a positive subsolution of $(1)-(2)$. For this, we let $\psi=\frac{1}{2} \rho \phi_{1}^{2}$. Note that $\|\psi\|_{\infty}<\rho$. Since $\nabla \psi=\rho \phi_{1} \nabla \phi_{1}$, a calculation shows that

$$
\begin{aligned}
-\Delta \psi & =-\frac{\rho}{2} \Delta \phi_{1}^{2} \\
& =-\rho\left(\left|\nabla \phi_{1}\right|^{2}+\phi_{1} \Delta \phi_{1}\right) \\
& =\rho\left(\lambda_{1} \phi_{1}^{2}-\left|\nabla \phi_{1}\right|^{2}\right) .
\end{aligned}
$$


Thus $\psi$ is a subsolution if

$$
\rho\left(\lambda_{1} \phi_{1}^{2}-\left|\nabla \phi_{1}\right|^{2}\right) \leq \lambda a(x) f(\psi)-c,
$$

Now $\lambda_{1} \phi_{1}^{2}-\left|\nabla \phi_{1}\right|^{2} \leq-k$ in $\bar{\Omega}_{\eta}$, and therefore

$$
\begin{aligned}
\rho\left(\lambda_{1} \phi_{1}^{2}-\left|\nabla \phi_{1}\right|^{2}\right) & \leq-k \rho \\
& \leq \lambda a(x) f(\psi)-c,
\end{aligned}
$$

if $c \leq c_{0}=k \rho$, since $\lambda a(x) f(\psi) \geq 0$.

Furthermore, we note that $\phi_{1} \geq \mu>0$ in $\Omega_{0}=\Omega \backslash \bar{\Omega}_{\eta}$, also in $\Omega_{0}$ we have

$$
\rho\left(\lambda_{1} \phi_{1}^{2}-\left|\nabla \phi_{1}\right|^{2}\right) \leq \lambda_{1} \rho
$$

Hence if

$$
\lambda \geq \lambda_{0}=\frac{\lambda_{1} \rho+c}{\alpha a_{0}},
$$

where $\alpha=\inf \left\{f(t): \frac{1}{2} \rho \mu^{2} \leq t \leq \frac{1}{2} \rho\right\}$, we have

$$
\begin{aligned}
\rho\left(\lambda_{1} \phi_{1}^{2}-\left|\nabla \phi_{1}\right|^{2}\right) & \leq \lambda_{1} \rho \\
& \leq \lambda a_{0} \alpha-c \\
& \leq \lambda a(x) f(\psi)-c .
\end{aligned}
$$

Therefore if $c \leq c_{0}$ and $\lambda \geq \lambda_{0}$, then $\psi$ is subsolution.

Next, we construct a supersolution $z$ of $(1)-(2)$ such that $z \geq \psi$. We denote $z=A \zeta(x)$, where the constant $A>0$ is large and to be chosen later. We shall verify that $z$ is a supersolution of $(1)-(2)$. A calculation shows that

$$
\begin{aligned}
-\Delta z & =A(-\Delta \zeta) \\
& =A .
\end{aligned}
$$

Thus $\psi$ is a subsolution if

$$
A \geq \lambda a(x) f(z)-c
$$

and therefore if $A \geq\|a\|_{\infty} A(\lambda)$ where $A(\lambda)=\sup _{[0, \rho]} f(t)$, we have

$$
-\Delta z \geq \lambda a(x) f(z)-c,
$$

and hence $z$ is supersolution of $(1)-(2)$. Since $\zeta>0$ and $\partial \zeta / \partial n<0$ on $\partial \Omega$, we can choose $A$ large enough so that $\psi \leq z$ is also satisfied. Hence Theorem 2.1 is proven. 


\section{$3 \quad$ Stability results}

In this section, we shall prove the instability of positive solution $u$ (obtained in Theorem 2.1 ) by showing that the principal eigenvalue $\mu_{1}$, of the equation linearized about $u$ is negative, the instability of $u$ then follows from the wellknown principle of linearized stability (see [8]).

We recall that, if $u$ be any nonnegative solution of

$$
\begin{cases}-\Delta u=g(x, u), & x \in \Omega, \\ u=0, & x \in \partial \Omega\end{cases}
$$

then the linearized equation about $u$ is

$$
\begin{cases}-\Delta \phi-g_{u}(x, u) \phi=\mu \phi, & x \in \Omega, \\ \phi=0, & x \in \partial \Omega .\end{cases}
$$

Definition 3.1. We call a solution $u$ of (6) a linearly stable solution if all eigenvalues of $(7)$ are strictly positive, which can be inferred if the principal eigenvalue $\mu_{1}>0$. Otherwise $u$ is linearly unstable.

Let $\mu_{1}$ be the principal eigenvalue of $(7)$ and $\psi$ be the corresponding eigenfunction. We make take $\psi$ such that $\psi>0$ in $\Omega$.

Our stability result is formulate in the following theorem.

Theorem 3.2. If $u \mapsto f(u) / u$ be strictly increasing, then the positive solution $u$ that obtained in Theorem 2.1 is linearly unstable.

Proof. Let $u$ be positive solution of (1) - (2) that obtained in Theorem 2.1, then from (7) the linearized equation about $u$ is

$$
\begin{gathered}
-\Delta \phi-\lambda a(x) f^{\prime} \phi=\mu \phi, \quad x \in \Omega, \\
\phi=0, \quad x \in \partial \Omega .
\end{gathered}
$$

Let $\mu_{1}$ be the principal eigenvalue and let $\psi(x)(\geq 0)$ be a corresponding eigenfunction. Multiplying (1) by $\psi(x)$ and (8) by $u$, then subtracting and integrating over $\Omega$, we obtain

$$
\begin{aligned}
\left.\int_{\Omega}[u \Delta \psi-\psi(x) \Delta u)\right] d x & +\lambda \int_{\Omega} a(x) \psi(x)\left[u f^{\prime}(u)-f(u)\right] d x \\
& +c \int_{\Omega} \psi(x) d x \\
& =-\mu_{1} \int_{\Omega} \psi(x) u(x) d x
\end{aligned}
$$


But by green's first identity

$$
\int_{\Omega} u \Delta \psi d x=-\int_{\Omega}(\nabla u \nabla \psi) d x
$$

and

$$
\int_{\Omega} \psi(x) \Delta u d x=-\int_{\Omega}(\nabla u \nabla \psi) d x .
$$

By using (11) - (12) in (10) we get

$$
-\mu_{1} \int_{\Omega} \psi(x) u(x) d x=\lambda \int_{\Omega} a(x) \psi(x)\left[u f^{\prime}(u)-f(u)\right] d x+c \int_{\Omega} \psi(x) d x .
$$

Our assumption implies that $u f^{\prime}(u)-f(u)>0$ for $u \in R^{+}$. Thus, we have

$$
-\mu_{1} \int_{\Omega} \psi(x) u(x) d x>0 .
$$

Hence, it is easy to see that $\mu_{1}<0$ and the result follows (see [8]).

\section{References}

[1] V. Anuradha, D.D. Hai and R. Shivaji, Existence results for superlinear semipositone boundary value problems, Proc. AMS, 124(3) (1996), 757763.

[2] H. Berestycki, A. Caffarelli, and L. Nirenberg, Further qualitative properties for elliptic equations in unbounded domains, Annali della Scuola Normale Superiore di Pisa. class di Scienze. Serie IV 25 (1997), no. 1-2, 69-94, dedicated to E. De Giorgi.

[3] K.J. Brown and R. Shivaj, Simple proofs of some resulte in perturbed bifurcation theort, Proc. Royal. Soc. Edinburgh. Sec A. Mathematics 93 (1982), no. 1-2, 71-82.

[4] A. Castro, S. Gadam and R. Shivaji, Evolution of Positive Solution Curves in Semipositone Problems with Concave Nonlinearities, Jour. Math. Anal. Appl., 245, (2000), 282-293. 
[5] P. Drabek and J. Hernandez, Existence and uniqueness of positive solutions for some quasilinear elliptic problem, Nonl. Anal, 44 (2001), no. 2, 189-204.

[6] D.D. Hai. On a class of sublinear quasilinear elliptic problems, Proc. Amer. Math. Soc, 131 (2003), no. 842409-2414.

[7] P.L. Lions, On the existence of positive solutions of semilinear elliptic equations, Siam Review, 24 (1982), 441-467.

[8] D.H. Satinger, Monotone methods in nonlinear elliptic and parabolic boundary value problems, Indiana Univ. Math. J., 21 (11)(1972), 9791000 .

Received: March 20, 2006 